\title{
Terapia de Reposição Hormonal e Qualidade da Vida Sexual de Mulheres no Climatério ${ }^{1}$
}

\author{
Regina Célia Souza Brito \\ Sheila Tetsume Makiama
}

Universidade Federal do Pará

\begin{abstract}
RESUMO
O climatério caracteriza-se pelo declínio gradual da produção de hormônios sexuais devido a perda da atividade folicular ovariana. Esta diminuição produz alterações físicas, como redução da elasticidade e da lubrificação vaginal, provocando desconforto durante a atividade sexual. Por isso, a Terapia de Reposição Hormonal (TRH) é considerada um importante tratamento para mulheres pré e pósmenopausadas. A proposta deste estudo foi avaliar a qualidade da vida sexual de mulheres entre $40 \mathrm{e}$ 60 anos e sua possível correlação com a prática de reposição hormonal. Noventa e duas mulheres nesta faixa etária, todas servidoras públicas com escolaridade igual ou superior ao nível médio completo, responderam voluntariamente a um questionário autoaplicável. Os dados foram coletados e analisados obedecendo às normas éticas de pesquisa com seres humanos. Encontrou-se uma possível correlação entre a idade e a falta de lubrificação vaginal. Entretanto, não houve diferença significativa entre a realização de terapia hormonal e a ausência de dificuldades sexuais. Infere-se que a qualidade da vida sexual não depende unicamente de variáveis fisiológicas, mas também de fatores sociais e conjugais. Sugere-se que mais estudos com amostra semelhante sejam realizados, assim como a realização de pesquisas acerca dos efeitos da ação conjunta de TRH e psicoterapia.

Palavras-chave: climatério; menopausa; terapia de reposição hormonal; qualidade de vida.
\end{abstract}

\begin{abstract}
Hormonal Replacement Therapy and Quality of Sex Life Among Women in Climateric

Climateric is characterized by a gradual decline of sexual hormones due the loss of ovarian follicular activity. The decrease produces physical changes, such as elasticity and vaginal lubrication reduction, causing discomfort during sexual intercourse. Therefore, Hormone Replacement Therapy (TRH) is considered an important treatment for pre and post menopausal women. This study aimed to assess sex life quality of women between 40 and 60 years of age and the possible correlation with hormone therapy. Ninety-two women, public workers, with high educational levels, voluntarily answered a selfreport questionnaire. Data were collected and analyzed following ethical standards for human research. A possible correlation was found between age and lack of vaginal lubrication. However, no significant relation was observed between hormone therapy and the absence of sexual difficulties. The results suggest that sex life quality maintenance is not only dependent on physiological variables but also on social and marital variables. It is suggested that more research with a similar sample should be conducted, as well as an evaluation of the effects of hormone therapy combined with psychotherapy.
\end{abstract}

Keywords: climacteric; menopause; hormone replacement therapy; quality of life.

O climatério, em uma perspectiva fisiológica, consiste na redução gradual da produção dos hormônios sexuais femininos devido à perda da atividade folicular ovariana. Esta perda paulatina é responsável pela diminuição da capacidade reprodutiva da mulher e progride até a interrupção da menstruação, mais ou menos por volta dos cinquenta anos de idade. O climatério compreende dois períodos: 1) a pré-menopausa, determinada por sangramento irregular com ou sem sintomas neurovegetativos, neuropsíquicos ou genitais e 2) a pós-meno-pausa, que teria como algumas das características a ausência de menstruação (amenorreia) há mais de um ano com ou sem sintomas (Marinho, Fernandes, Wehba, Pinto Neto \& Baracat, 2001). A menopausa consiste na última menstruação da mulher, delimitando as duas fases do climatério (Barini, 2001). 
Através de diálogos com essas mulheres chegou-se à conclusão de que, para elas, a vida sexual não termina (ou pelo menos elas não gostariam que terminasse) quando o climatério começa. Um estudo canadense mostrou que uma parcela considerável de mulheres que estava na fase de transição ou na pós-menopausa, considerava o sexo um fator importante para manter a qualidade de vida, assim como a qualidade do relacionamento (intimidade e respeito) para a satisfação sexual (Basson, 2006). Já em um estudo publicado por Mishra e Kuh (2006), menos de um terço de 1.525 participantes relataram declínio da atividade sexual comparado ao ano anterior à menopausa, e menos de $20 \%$ declararam dificuldades durante a cópula. Neste estudo, o declínio na vida sexual foi associado a sintomas psicológicos e ao stress, enquanto que a dificuldade no coito esteve mais associada a sintomas somáticos e vasomotores. O estudo de Basson (2006) identificou fatores que contribuiriam para resiliência de problemas sexuais nesta fase, sendo os mais citados: sentimentos positivos pelo parceiro, saúde mental, boa interação sexual com o parceiro e experiências sexuais anteriores positivas.

Um estudo comparativo sobre a qualidade de vida entre usuárias e não usuárias de Terapia de Reposição Hormonal (TRH) que frequentavam o ambulatório de climatério de uma universidade no estado do Rio de Janeiro não encontrou diferença estatisticamente significativa entre as amostras, apesar das usuárias de TRH terem relatado menor frequência de sintomas de intensidade moderada e acentuada, característicos do climatério (Zahar, Aldrighi, Neto, Conde, Zahar e cols., 2005). Em outro estudo brasileiro, agora com mulheres usuárias de TRH em um ambulatório de climatério de uma universidade do sudeste, foi considerada comprometida a qualidade de vida da amostra estudada, principalmente nos quesitos de sintomas somáticos, humor deprimido e ansiedade. Este estudo também considerou que a TRH não estava associada à qualidade de vida, concluindo que tanto fatores biológicos quanto culturais e psicossociais são importantes para a aquisição e/ou manutenção da mesma (Lorenzi, Baracat, Saciloto \& Padilha, 2006). Entretanto, outro estudo concluiu como significativa a relação entre TRH e a melhora de sintomas climatéricos e da qualidade de vida em comparação com grupo placebo (Wirklund, Karlberg \& Mattsson, 1993) e entre o TRH e a melhora da função sexual do orgasmo, lubrificação e dispareunia (dor no coito) (González, Viáfara \& Molina, 2004; Vigeta \& Brêtas, 2004).
Com o avanço da idade ocorre uma redução quantitativa da resposta sexual em decorrência da diminuição na produção dos hormônios sexuais, o que produz alterações físicas, como diminuição da elasticidade e da lubrificação da vagina. Por isso, a TRH - que consiste na prática de administração de hormônios sexuais (e.g. estrógeno) através de via oral, transdérmica ou percutânea - começou a ser indicada como tratamento ideal para as mulheres no climatério. Esta reposição hormonal, além de restabelecer o controle vasomotor e tratar das "ondas de calor" (fogachos), aumenta o fluxo sanguíneo, prevenindo a falta de lubrificação e diminuindo a dispareunia (Bachmann \& Nevadunsky, 2000; Lopes \& Maia, 1994), além de tratar das alterações no trato urinário (Bachmann \& Nevadunsky, 2000). Entretanto, vale ressaltar que o uso prolongado de estrógeno combinado com progesterona pode duplicar os riscos de incidência de câncer de mama quando comparado a um grupo de não usuárias (Chlebowski, Kuller, Prentice, Stefanick, Manson, Gass e cols., 2009). Por conseguinte, segundo Wannmacher e Lubianca (2004), a indicação de TRH deve limitarse exclusivamente ao controle de sintomas climatéricos e, preferencialmente, por curto prazo.

Não obstante, apesar de algumas dificuldades sexuais resultarem de desequilíbrios hormonais - tendo, portanto, causa fisiológica - não se pode ignorar a ação de fatores psicossociais tais como: grau de satisfação no relacionamento conjugal, intimidade, respeito, representação social da menopausa tanto para a mulher quanto para o parceiro (Basson, 2006). Segundo Lopes e Maia (1994), em alguns casos, a mulher apresentaria redução de sua resposta sexual devido à doença ou declínio do interesse do cônjuge. Goberna e cols. (2009) concluíram em seu estudo que a diminuição da atividade sexual não é inerente a essa fase, mas sim dependente de fatores como o bem-estar, tanto da mulher quanto do seu parceiro. Nesta pesquisa espanhola, verificou-se também que o convívio com os filhos e os pais idosos na mesma residência emergiu como um fator que contribuiria para a diminuição do desejo e da prática sexual do casal. Em outro estudo, concluiu-se que sintomas psicológicos, como nervosismo e depressão, aumentariam as experiências de dispareunia (Valadares, Pinto-Neto, Conde, Sousa, Osis \& Costa-Paiva, 2008). Assim, variáveis psicossociais podem interferir mais na determinação do comportamento sexual da mulher do que a ação de hormônios (Dennerstein, Dudley \& Burger, 2001; Dennerstein, Lehert, \& Burger, 2005; Goberna e cols., 2009; Woods, Mitchell, Percival \& Smith-DiJulio, 2009), sendo 
então aconselhável, em casos de real diminuição de desejo, não somente a reposição de hormônios, mas também a realização de aconselhamento e reeducação sexual (Lopes \& Maia, 1994). A inibição do desejo ou falta de interesse pelo sexo - por exemplo, poderia ser produto não unicamente do declínio na produção de hormônios, mas também do deterioramento da relação conjugal.

Faz-se também necessário ressaltar aqui algumas características que permeiam os estudos brasileiros sobre qualidade de vida e a reposição hormonal. As amostras estudadas, de modo geral, são de mulheres que estão em tratamento em ambulatórios de climatério em hospitais universitários, o que permite pressupor a preexistência de queixas, já que as mesmas procuraram atendimento médico. Essas participantes, na sua maioria, possuem baixa escolaridade e são economicamente carentes. Este conjunto de características pode confundir os resultados uma vez que se torna difícil isolar as condições nutricionais e outras patologias precedentes. Isso somado aos anos de escolaridade que podem influenciar na quantidade e qualidade de informações necessárias que essas mulheres obtêm para aderir ao tratamento hormonal.

A importância de pesquisas com mulheres com bom nível de escolaridade e renda, e sem queixas precedentes (sem patologias) consiste na obtenção de dados que possam clarear as demais variáveis envolvidas. Assim, o objetivo deste estudo foi avaliar, através de aplicação de questionário com mulheres no climatério, se mudanças na resposta sexual seriam estritamente oriundas da diminuição da produção de hormônios sexuais - o que seria resolvido com TRH ou se fatores psicossociais contribuiriam para esta mudança.

\section{MÉTODO}

\section{Participantes}

Participaram deste estudo 92 mulheres, com idade entre 40 e 60 anos de idade, todas servidoras públicas de uma instituição de ensino federal com nível de escolaridade igual ou superior ao nível médio completo e renda mensal igual ou superior a quinhentos reais.

\section{Instrumento e Material}

Para este estudo, foram selecionadas e examinadas questões acerca da idade, reposição hormonal (se faz ou não), frequência da prática sexual, grau de satisfa- ção no relacionamento e desempenho sexual do parceiro atual, além da referência a dificuldades na atividade sexual, questões estas contidas em um questionário autoaplicável. Todas as questões, exceto sobre idade, reposição hormonal e frequência da prática sexual eram de múltipla escolha. Foram também utilizados: dois Termos de Consentimento Livre e Esclarecido, um envelope e um adesivo para lacrar o envelope no momento de devolução do mesmo.

\section{Procedimento}

As participantes foram escolhidas aleatoriamente através de lista de servidores públicos fornecida pelo setor de Recursos Humanos da instituição e abordadas em seu local de trabalho por um(a) pesquisador(a). A participante era questionada sobre a possibilidade de participar da pesquisa e um dos Termos de Consentimento era lido. Quando confirmada a participação, os dois termos eram assinados pela participante, um era devolvido à pesquisadora e o outro era entregue juntamente com o questionário à participante, ficando estabelecido o dia, hora e local para a devolução do último.

Os dados foram coletados e analisados obedecendo-se as normas éticas para pesquisa com humanos. As análises estatísticas (percentuais e crosstabs) dos dados coletados foram realizadas através do programa SPSS 11.5 for Windows.

\section{RESULTADOS}

As mulheres participantes tinham, em média, 48 anos, sendo que $58,7 \%$ tinham idade na pré-menopausa (de 40 a 49 anos) e $41,3 \%$, na pós-menopausa (de 50 a 60 anos).

Entre as participantes de 40 a 49 anos, $81,5 \%$ declararam que mantiveram vida sexualmente ativa no último ano e, atualmente, $26,2 \%$ praticam sexo pelo menos 2 vezes por semana, apesar de 31,8\% terem relatado que gostariam de fazer sexo 4 vezes por semana. Entre as participantes de 50 a 60 anos, $74 \%$ declararam que mantiveram vida sexualmente ativa no último ano e, atualmente, $31 \%$ praticam sexo 2 vezes por semana, sendo esta a quantidade desejada por $22,9 \%$ das participantes.

Observou-se que apenas $15 \%$ das participantes com menos de 50 anos declarou que fazia TRH, enquanto que $47 \%$ das participantes na pós-menopausa já a fazia. Nas Tabelas 1 e 2, está demonstrado o percentual das dificuldades sexuais mais apontadas pelas participantes. 
TABELA 1

Percentual de Mulheres que Apontaram Dor na Relação Sexual e a Falta de Desejo Sexual Como as Dificuldades Sexuais Mais Frequentes

\begin{tabular}{lccccccc}
\hline & & \multicolumn{3}{c}{ Dor na relação sexual } & \multicolumn{3}{c}{ Falta de desejo sexual } \\
& & Sim & Não & Total & Sim & Não & Total \\
\hline Situação da & Pré-menopausa & $5,6 \%$ & $94,4 \%$ & $100 \%$ & $25,9 \%$ & $74,1 \%$ & $100 \%$ \\
Participante & Pós-menopausa & $13,2 \%$ & $86,8 \%$ & $100 \%$ & 31,6 & 68,4 & $100 \%$ \\
\hline
\end{tabular}

TABELA 2

Percentual de Mulheres que Apontaram a Falta de Lubrificação e a Dificuldade Para Atingir o Orgasmo Como as Dificuldades Sexuais Mais Frequentes

\begin{tabular}{cccccccc}
\hline & & \multicolumn{2}{c}{ Falta de } & \multicolumn{3}{c}{ Dificuldade para } \\
& & \multicolumn{2}{c}{$\begin{array}{c}\text { lubrificação } \\
\text { atingir o orgasmo }\end{array}$} & Total & Total \\
& Sim & Não & & Sim & Não & \\
\hline Situação da & Pré-menopausa & $16,7 \%$ & $83,3 \%$ & $100 \%$ & $18,5 \%$ & $81,5 \%$ & $100 \%$ \\
Participante & Pós-menopausa & $42,1 \%$ & $57,9 \%$ & $100 \%$ & $28,9 \%$ & $71,1 \%$ & $100 \%$ \\
\hline
\end{tabular}

Dentre as mulheres na pré-menopausa, a falta de desejo sexual e a dificuldade para atingir o orgasmo ( $26 \%$ e $18,5 \%$ respectivamente) foram as dificuldades sexuais mais apontadas, enquanto que, entre as mulheres na pós-menopausa, a falta de lubrificação $(42,1 \%)$ e a falta de desejo $(31,6 \%)$ foram as dificuldades mais citadas. Através dos percentuais comparados por idade, verificou-se que idade e falta de lubrificação podem estar correlacionadas.

$\mathrm{Na}$ Tabela 3, está representado o percentual de participantes que fazia TRH e que apresentam dor durante a cópula (dispareunia).

TABELA 3

Percentual de Mulheres que Apresentavam Dispareunia e que Faziam TRH

\begin{tabular}{|c|c|c|c|c|c|}
\hline & & & \multicolumn{2}{|c|}{ Reposição Hormonal } & \multirow[b]{2}{*}{ Total } \\
\hline & & & Sim & Não & \\
\hline \multirow{4}{*}{ 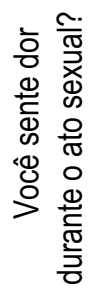 } & \multirow{2}{*}{ Sim } & Pré-menopausa & $0 \%$ & $100 \%$ & $100 \%$ \\
\hline & & Pós-menopausa & $40 \%$ & $60 \%$ & $100 \%$ \\
\hline & \multirow{2}{*}{ Não } & Pré-menopausa & $15,7 \%$ & $84,3 \%$ & $100 \%$ \\
\hline & & Pós-menopausa & $48,5 \%$ & $51,5 \%$ & $100 \%$ \\
\hline
\end{tabular}

Nos dados apresentados na Tabela 3, observou-se que não houve diferença relevante entre a realização de TRH e presença de dor durante o coito. Dentre as mulheres pré-menopausadas que relataram sentir dor durante a cópula, nenhuma fazia TRH. Entretanto, dentre as mulheres nesta mesma fase que relataram não sentir dor no coito, a maioria $(84,3 \%)$ não fazia RH. Dentre as mulheres pós-menopausadas que acusaram dispareunia como uma dificuldade, $60 \%$ não faziam
TRH, sendo que, dentre as mulheres nessa fase que relataram não sentir dor durante o coito, $51,5 \%$ declarou que não faziam TRH. Verificou-se um percentual equilibrado entre a ausência de dor durante o coito tanto entre mulheres que faziam e que não faziam TRH. Pôde-se inferir através desse resultado que, para as participantes deste estudo, a TRH não foi uma variável que interferiu significativamente na prevenção ou solução dessa disfunção sexual. 
Na Tabela 4 apresenta-se o percentual de partici- desejo sexual. pantes que faziam TRH e que apresentavam falta de

TABELA 4

Percentual de Mulheres que Apresentavam Falta de Desejo Sexual e que Faziam TRH

\begin{tabular}{|c|c|c|c|c|c|}
\hline & & & \multicolumn{2}{|c|}{ Reposição Hormonal } & \multirow[t]{2}{*}{ Total } \\
\hline & & & Sim & Não & \\
\hline \multirow{4}{*}{ 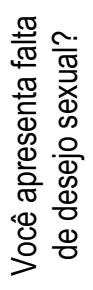 } & \multirow{2}{*}{ Sim } & Pré-menopausa & $7,1 \%$ & $92,9 \%$ & $100 \%$ \\
\hline & & Pós-menopausa & $41,7 \%$ & $58,3 \%$ & $100 \%$ \\
\hline & \multirow{2}{*}{ Não } & Pré-menopausa & $17,5 \%$ & $82,5 \%$ & $100 \%$ \\
\hline & & Pós-menopausa & $50 \%$ & $50 \%$ & $100 \%$ \\
\hline
\end{tabular}

A partir dos dados ilustrados na Tabela 4, observou-se que não houve grande diferença entre a realização de TRH e a falta de desejo sexual. Dentre as participantes na pré-menopausa que relataram falta de desejo sexual, 7,1\% faziam TRH. Entretanto, dentre as participantes nessa mesma fase que relataram ainda possuir desejo sexual, a maioria $(82,5 \%)$ não fazia TRH. Dentre as mulheres na pós-menopausa que relataram a falta de desejo, 58,3\% não faziam RH, sendo que, dentre as 26 mulheres nesse período que rela- taram sentir desejo sexual, $50 \%$ não fazia TRH. Mais uma vez, observou-se um percentual equilibrado entre o desejo sexual e a realização/não realização de TRH. Pôde-se inferir através desse resultado que, para as participantes deste estudo, a reposição hormonal não foi uma variável influente na manutenção ou aumento do desejo sexual.

$\mathrm{Na}$ Tabela 5 verifica-se o percentual de participantes que fazia TRH e que apresentavam falta de lubrificação vaginal.

TABELA 5

Percentual de Mulheres que Apresentavam Falta de Lubrificação Vaginal e que Faziam TRH

\begin{tabular}{|c|c|c|c|c|c|}
\hline & & & \multicolumn{2}{|c|}{ Reposição Hormonal } & \multirow[t]{2}{*}{ Total } \\
\hline & & & Sim & Não & \\
\hline \multirow{4}{*}{ 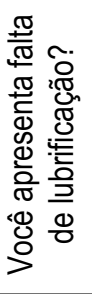 } & \multirow{2}{*}{ Sim } & Pré-menopausa & $33,3 \%$ & $66,7 \%$ & $100 \%$ \\
\hline & & Pós-menopausa & $43,8 \%$ & $56,3 \%$ & $100 \%$ \\
\hline & \multirow{2}{*}{ Não } & Pré-menopausa & $11,1 \%$ & $88,9 \%$ & $100 \%$ \\
\hline & & Pós-menopausa & $50 \%$ & $50 \%$ & $100 \%$ \\
\hline
\end{tabular}

Das participantes na pré-menopausa que relataram falta de lubrificação vaginal, 33,3\% faziam TRH. Já dentre as 45 participantes nesse mesmo período que relataram não ter problema com a lubrificação, a maioria $(88,9 \%)$ não fazia TRH. E aquelas na pós-menopausa que relataram a falta de lubrificação, $56,3 \%$ não faziam TRH, sendo que, dentre as 22 mulheres nessa fase que não relataram a falta de lubrificação como uma dificuldade, $50 \%$ não faziam TRH. Outra vez, observou-se um percentual equilibrado entre a realiza- ção de TRH e a ausência/presença de lubrificação vaginal. Através desse dado pode-se supor que, para as participantes deste estudo, a reposição hormonal ou foi uma variável que influenciou, embora em menor grau na lubrificação da vagina, ou outras variáveis, como a idade da participante, interferiram neste fator.

Com relação à dificuldade para atingir o orgasmo, na Tabela 6 verifica-se o percentual de participantes que fazia TRH e que apresentam esta disfunção. 
TABELA 6

Percentual de Mulheres que Apresentavam Dificuldade Para Atingir o Orgasmo e que Faziam TRH

\begin{tabular}{|c|c|c|c|c|c|}
\hline & & & \multicolumn{2}{|c|}{ Reposição Hormonal } & \multirow[t]{2}{*}{ Total } \\
\hline & & & Sim & Não & \\
\hline \multirow{4}{*}{ 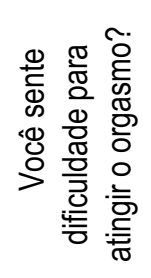 } & \multirow{2}{*}{ Sim } & Pré-menopausa & $10 \%$ & $90 \%$ & $100 \%$ \\
\hline & & Pós-menopausa & $45,5 \%$ & $54,5 \%$ & $100 \%$ \\
\hline & \multirow{2}{*}{ Não } & Pré-menopausa & $15,9 \%$ & $84,1 \%$ & $100 \%$ \\
\hline & & Pós-menopausa & $48,1 \%$ & $51,9 \%$ & $100 \%$ \\
\hline
\end{tabular}

Na Tabela 6 observa-se que as participantes na prémenopausa, que relataram dificuldade para atingir o orgasmo, 90\% não fazia TRH. Entretanto, dentre as participantes nessa mesma fase que relataram não ter dificuldade para atingir o orgasmo, a maioria $(84,1 \%)$ também não faz TRH. Dentre as mulheres na pósmenopausa que relataram a dificuldade para atingir o orgasmo, $54,5 \%$ não faziam $\mathrm{RH}$, sendo que, dentre as mulheres nessa mesma fase que relataram não sentir dificuldade para obter orgasmo, 51,9\% não fazia TRH. Observou-se mais uma vez um percentual equilibrado entre a realização de TRH e a ausência/presença de dificuldade para atingir o orgasmo. Com esse resultado pode-se inferir que, para as participantes deste estudo, a reposição hormonal não se constituiu como uma variável que influencia significativamente na obtenção de orgasmo, havendo outras variáveis envolvidas.

Em função destes resultados serão analisados aspectos psicossociais que possam ter interferido nas análises sobre a importância do TRH para a manutenção de qualidade de vida sexual entre mulheres de 40 a 60 anos de idade.

$\mathrm{Na}$ Tabela 7, estão representados os percentuais do grau de atratividade do parceiro atual relacionados aos percentuais de falta de desejo.

TABELA 7

Percentuais de Grau de Atratividade do Parceiro e a Falta de Desejo Sexual

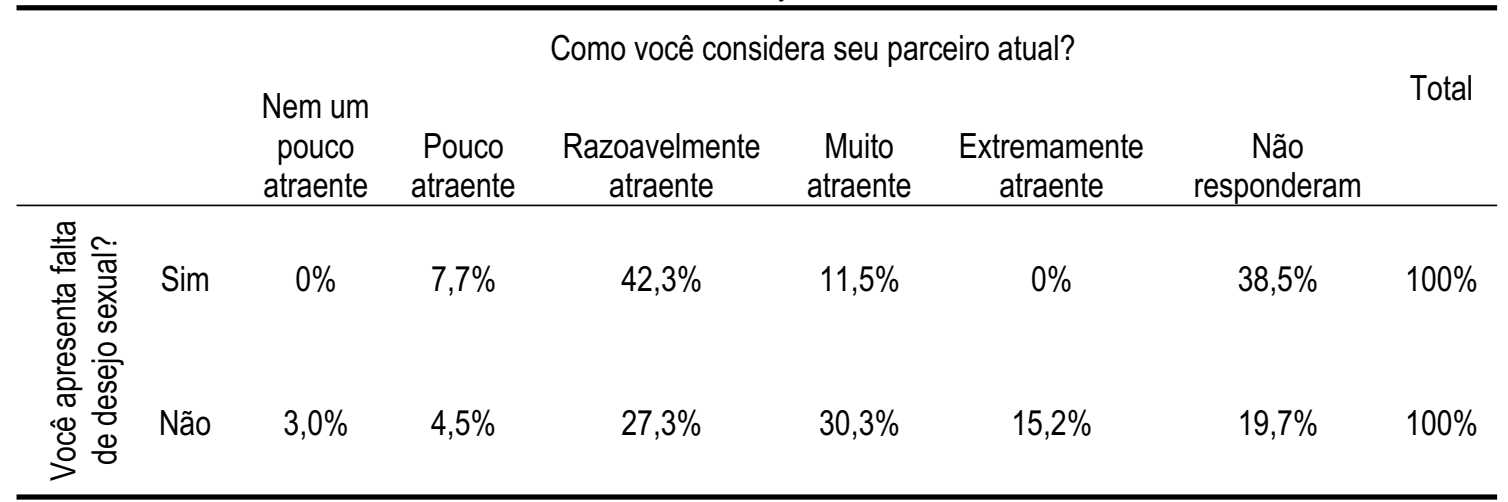

A partir dos dados mostrados na Tabela 7, pôde-se inferir que há uma relação entre a atratividade do parceiro e a manutenção de desejo sexual. Das mulheres que se declararam não tendo problemas de desejo sexual $72,8 \%$ delas avaliaram seus parceiros nas categorias Razoavelmente, Muito e Extremamente Atraente e apenas 19,7\% não responderam. Já juntando os percentuais de atratividade dos parceiros das mulheres que afirmam ter falta de desejo verifica-se que apenas $53,8 \%$ declararam que consideravam seus parceiros atuais como Razoavelmente e Muito atraente, sendo a maioria delas $42,3 \%$ os considerou Razoavelmente Atraente. Nenhuma delas os considerou Extremamente Atraente e, sintomaticamente $38 \%$ não responderam à questão. Assim, é forte o indício de que a atratividade do parceiro pode ser uma variável rele- 
vante na manutenção do desejo sexual nesta fase da vida.

Verificou-se então se a avaliação positiva do desempenho sexual do parceiro também estava relacio- nada com a falta de desejo sexual. Na Tabela 8 , estão representados os percentuais do grau de satisfação com desempenho sexual do parceiro atual relacionados aos percentuais de falta de desejo.

TABELA 8

Percentuais de Grau de Satisfação com Desempenho Sexual do Parceiro Atual e a Falta de Desejo Sexual

\begin{tabular}{|c|c|c|c|c|c|c|c|}
\hline & & \multicolumn{5}{|c|}{ Qual o grau de satisfação com o desempenho sexual do seu parceiro atual? } & \multirow[t]{2}{*}{ Total } \\
\hline & & Baixo & Razoável & Bom & Muito Bom & Não responderam & \\
\hline 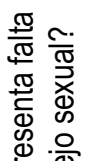 & Sim & $3,8 \%$ & $19,2 \%$ & $19,2 \%$ & $15,4 \%$ & $42,3 \%$ & $100 \%$ \\
\hline 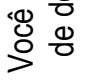 & Não & $4,5 \%$ & $3 \%$ & $36,4 \%$ & $33,3 \%$ & $22,7 \%$ & $100 \%$ \\
\hline
\end{tabular}

Aqui também se encontrou uma relação entre o grau de satisfação com o desempenho sexual do parceiro atual e a falta de desejo sexual, haja vista que $33,3 \%$ e $36,4 \%$ das mulheres que relataram não ter problema relacionado ao desejo sexual consideraram o desempenho do parceiro "muito bom" e "bom", respectivamente, comparado aos $15,4 \%$ e $19,2 \%$ de mulheres que apontaram a falta de desejo sexual como uma dificuldade presente, apesar de considerar o desempenho do parceiro "muito bom" e "bom", respectivamente. Já 19,2\% de participantes que relataram ter problema relacionado ao desejo sexual, consideraram o desempenho do seu parceiro atual "razoável", comparado aos $2 \%$ de mulheres que não relataram a falta de desejo sexual como uma dificuldade presente, mas que consideraram o desempenho sexual do parceiro atual "razoável"; $28,3 \%$ das participantes não responderam ou porque não quiseram ou porque não possuíam parceria até a data da coleta de dados.

$\mathrm{Na}$ Tabela 9, estão representados percentuais do grau de satisfação com desempenho sexual do parceiro atual relacionados aos percentuais de dificuldade para atingir o orgasmo.

TABELA 9

Percentuais de Grau de Satisfação com Desempenho Sexual do Parceiro Atual e Dificuldade Para Atingir o Orgasmo

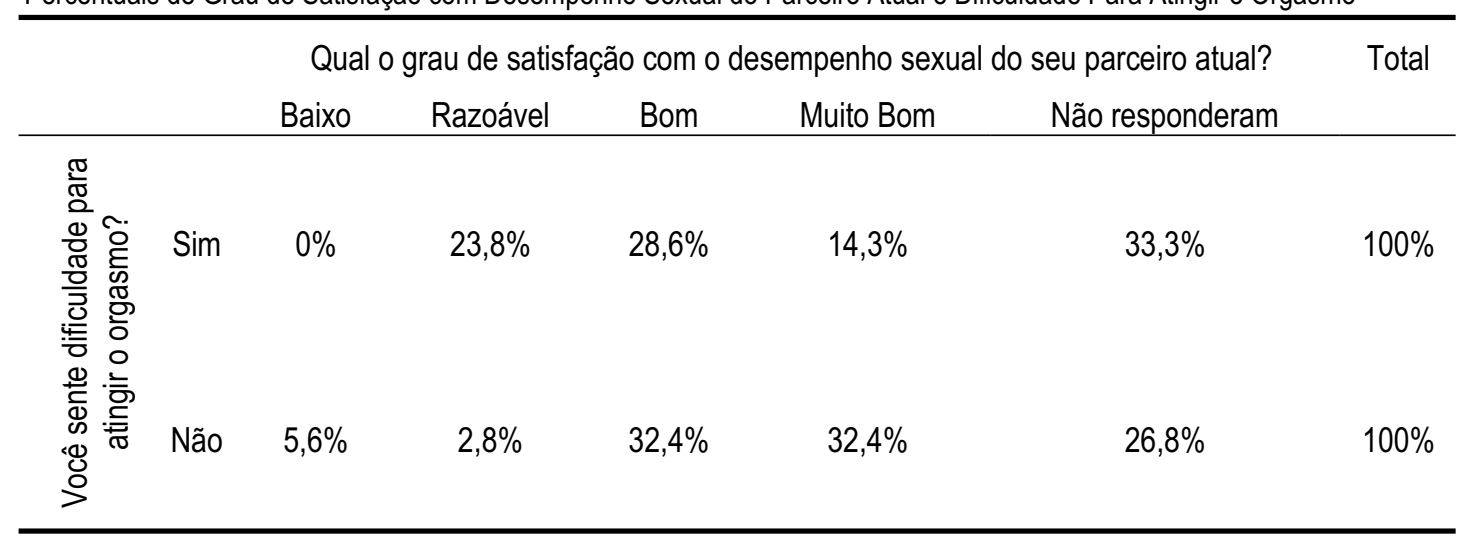

Outra vez pode-se inferir uma relação entre o grau de satisfação com o desempenho sexual do parceiro atual e a dificuldade para atingir o orgasmo. 32,4\% das mulheres que relataram não apresentar dificuldade para ter orgasmo consideraram o desempenho do parceiro "muito bom", comparado aos 14,3\% de mulheres que apontaram essa dificuldade, apesar de considerar o desempenho do parceiro "muito bom". Já 23,8\% 
de participantes que relataram ter problema para atingir o orgasmo, consideraram o desempenho do seu parceiro atual "razoável", comparado aos $2,8 \%$ de mulheres que não relataram essa dificuldade e consideraram o desempenho sexual do parceiro atual "razoável"; $28,3 \%$ das participantes não responderam ou porque não quiseram ou porque não possuem parceria atual. A dor no coito e a falta de lubrificação não apresentaram percentuais que indicassem relação com o desempenho sexual do parceiro atual e, por isso, não foram aqui ilustradas.

\section{DISCUSSÃO}

Com base nos dados desse estudo, ratificou-se que a obtenção e manutenção da qualidade da vida sexual humana são multideterminadas, não dependendo unicamente de variáveis fisiológicas, mas também de fatores sociais e conjugais. O significado da menopausa para a sociedade, incluindo para a própria mulher $\mathrm{e}$ seu companheiro, reflete a forma como a mulher lida com essa fase não reprodutiva. Esta nova fase pode ser associada, nas sociedades ocidentais, tanto ao fim das restrições e preocupações relacionadas à concepção e aumento da segurança e experiência, quanto pode ser associada à interrupção da feminilidade e término da beleza e juventude (Uchôa, 2003; Collyer, 2005). Através dos dados desse estudo pôde-se inferir que variáveis como atratividade e grau de satisfação com o desempenho sexual do parceiro exercem influência positiva ou negativa na manutenção de uma vida sexual com qualidade, e não apenas o declínio da produção de hormônios sexuais. Assim, os resultados aqui apresentados reforçam a hipótese de que fatores psicossociais, como a parceria afetiva e a satisfação conjugal, apresentada por Dennerstein e cols. (2005) e Goberna e cols. (2009), atuam no comportamento sexual a ponto de comprometer ou melhorar a qualidade da vida sexual, assim como as mudanças hormonais características da menopausa. Estes resultados fortalecem também os achados de Lorenzi (2006) e de Zahar e cols. (2005) de que a TRH pode não estar diretamente associada à qualidade de vida de mulheres na menopausa.

Além disso, é importante municiar as mulheres que estão vivenciando esta fase do seu ciclo vital com informações que permitam a elas decidir com menos pressão se querem ou não aderir a TRH. É claro que se elas forem informadas claramente da função da TRH e da função das outras variáveis envolvidas na questão da qualidade e bem-estar de suas vidas sexuais, elas estarão mais habilitadas a fazer escolhas mais realistas.

Entretanto, faz-se necessário relatar que, devido às questões aqui analisadas estarem inseridas em um grande questionário exploratório e não exigir respostas das participantes em escalas numéricas como a Likert, por exemplo, a análise dos dados ficou incompleta, impossibilitando as análises de significância estatística. Não obstante, considerou-se que os dados apresentados são válidos e importantes em função das características da amostra aqui investigada.

\section{REFERÊNCIAS}

Bachmann. G, A., \& Nevadunsky, N. S. (2000). Diagnosis and treatment of atrophic vaginitis. American Family Physician, 61(10), 3090-3096.

Barini, R. (2001). Modificações hormonais e variações comportamentais na mulher. Em D. R. Zamignani (Org.). Sobre comportamento e cognição: Vol. 3. A aplicação da análise do comportamento e da terapia cognitivo-comportamental no hospital geral e nos transtornos psiquiátricos (pp. 45-52). Santo André, SP: ESETec .

Basson, R. (2006). The complexities of women's sexuality and the menopause transition. Menopause, 13(6), 853-855.

Chlebowski, R. T., Kuller, L. H., Prentice, R. L., Stefanick, M. L., Manson, J. E., Gass, M. e cols. (2009). Breast cancer after use of estrogen plus progestin in postmenopausal women. New England Journal of Medicine, 360, 573-587.

Collyer, S. C. (2005). Relatos da atividade sexual de mulheres na pós-menopausa. Dissertação de Mestrado não-publicada, Universidade Federal do Pará, Belém.

Dennerstein, L., Dudley, E., \& Burger H. (2001). Are changes in sexual functioning during midlife due to aging of menopause? Fertility and Sterility, 76(3), 456-460.

Dennerstein, L., Lehert P., \& Burger, H. (2005). The relative effects of hormones and relationship factors on sexual function of women through the natural menopausal transition. Fertility and Sterility, 84(1), 174-180.

Goberna, J., Francés, L., Paulí, A., Barluenga, A., \& Gascón, E. (2009). Sexual experiences during the climacteric years: What do women think about it? Maturitas, 62(1), 47-52.

González, M., Viáfara, G., Caba, F., \& Molina, E. (2004). Sexual function, menopause and hormone replacement therapy (HRT). Maturitas, 48(4), 411-420. Retirado em 28 de fevereiro de 2007, da base de dados ScienceDirect.

Lopres, G., \& Maia, M. (1994). Sexualidade e envelhecimento ( $3^{\mathrm{a}}$ ed.). São Paulo: Saraiva.

Lorenzi, D. R.. S., Baracat, E. C., Saciloto, B., \& Padilha, I. (2006). Fatores associados à qualidade de vida após a menopausa. Revista da Associação Médica Brasileira, 52(5), 312317. Retirado em 28 de fevereiro de 2007, da base de dados SciELO.

Marinho, R. M., Fernandes, C. E., Wehba, S., Pinto Neto, A. M., $\&$ Baracat, E. C. (2001). Atenção primária e terapia de reposi- 
ção hormonal no climatério. Retirado em 05 de setembro de 2007, de http://www.projetodiretrizes.org.br/projeto_diretrizes/ 034.pdf.

Misha, G., Kuh, D. (2006). Sexual functioning through menopause: The perceptions of women in a British cohort. Menopause, 13(6), 880-890.

Uchoa, E. (2003). Contribuições da antropologia para uma abordagem das questões relativas à saúde do idoso. Cadernos de Saúde Pública, 19, 849-853.

Valadares, A. L., Pinto-Neto, A. M., Conde, D. M., Sousa, M. H., Osis M. J., \& Costa-Paiva, L. (2008). A population-based study of dyspareunia in a cohort of middle-aged Brazilian women. Menopause, 15(6), 1184-1190.

Vigeta, S. M. G., \& Brêtas, A. C. P. (2004). A experiência da perimenopausa e pós-menopausa com mulheres que fazem uso ou não da terapia de reposição hormonal. Cadernos de Saúde Pública, 20, 1682-1689.

Zahar, S. E. V., Aldrighi, J. M., Neto, A. M. P., Conde, D. M., Zahar, L. O., \& Russomano, F. (2005). Qualidade de vida em usuárias e não usuárias de terapia de reposição hormonal. Re- vista da Associação Médica Brasileira, 51(3), 133-138. Retirado em 28 de fevereiro de 2007, da base de dados SciELO.

Wannmacher, L., \& Lubianca, J. N. (2004). Terapia de reposição hormonal na menopausa: Evidências atuais. Retirado em 25 de fevereiro de 2009, de http://bvsms.saude.gov.br/bvs/publicacoes/HSE_URM_TRH_0504.pdf.

Wiklund, I., Karlberg, J., \& Mattsson, L. A. (1993). Quality of life of postmenopausal women on a regimen of transdermal estradiol therapy: A double-blind placebo-controlled study. American Journal of Obstetrics and Gynecology, 168, 824-830.

Woods, N. F., Mitchell, E. S., Percival, D. B., \& Smith-DiJulio, K. (2009). Is the menopausal transition stressful? Observations of perceived stress from the Seattle Midlife Women's Health Study. Menopause, 16(1), 90-97.

\footnotetext{
Notas:

${ }^{1}$ Trabalho Financiado pelo CNPq: Instituto do Milênio / 420038 / 2005.

${ }^{2}$ Mestranda bolsista do CNPq.
}

\section{Sobre as autoras:}

Regina Célia Souza Brito: Doutora em Psicologia Experimental. Programa de Pós-graduação em Teoria e Pesquisa do Comportamento - Universidade Federal do Pará. Endereço eletrônico: rcsb@uol.com.br.

Sheila Tetsume Makiama: Mestranda do Programa de Pós-graduação em Teoria e Pesquisa do Comportamento - Universidade Federal do Pará. Endereço eletrônico: sheilamakiama@ hotmail.com.

Endereço para correspondência: Sheila Tetsume Makiama - Travessa Angustura, 3.829 - Bloco 3 - apto. 101 - 66095-040 Belém - PA 